\title{
Synthesis and Evaluation of 2-Hydroxy-1,4-naphthoquinone Derivatives as Potent Antimalarial Agents
}

\author{
Wanthani Paengsri, ${ }^{a, b}$ Napapha Promsawan, ${ }^{* a}$ and Apiwat Baramee ${ }^{a}$ \\ ${ }^{a}$ Department of Chemistry, Faculty of Science, Chiang Mai Universit; Chiang Mai 50200, Thailand: and ${ }^{b}$ Center of \\ Excellence for Innovation in Chemistry, Faculty of Science, Chiang Mai University; Chiang Mai 50200, Thailand. \\ Received September 24, 2020; accepted December 24, 2020; advance publication released online January 8, 2021
}

\begin{abstract}
A series of 3-substituted-2-hydroxy-1,4-naphthoquinone derivatives with a variety of side chains were successfully synthesized by Mannich reaction of 2-hydroxy-1,4-naphthoquinone (lawsone) with selected amines and aldehydes. All substances (1-16) were evaluated for in-vitro antimalarial activity against strains of Plasmodium falciparum by microculture radioisotope technique. Bioassay data revealed that ten derivatives (1-8, 11 and 13) displayed significantly good activity with values of $\mathrm{IC}_{50}$ ranging from 0.77 to $4.05 \mu \mathrm{g} / \mathrm{mL}$. The best biological profile $\left(\mathrm{IC}_{50}=0.77 \mu \mathrm{g} / \mathrm{mL}\right.$ ) was observed in compound 1 , possessing a $n$-butyl substituted aminomethyl group. Experimental results support the potential use of our active Mannich components as promising antimalarial agents in the fight against malaria infections and multidrug resistance problems.
\end{abstract}

Key words lawsone; Mannich reaction; antimalarial activity; Plasmodium falciparum

\section{Introduction}

Malaria is an ancient infectious disease affecting humans and animals which is transmitted by infected female Anopheles mosquitoes. Parasitic protozoa from the genus Plasmodium is known to be the cause of the infection. There are five different Plasmodium species, Plasmodium falciparum, Plasmodium vivax, Plasmodium ovale, Plasmodium knowlesi and Plasmodium malariae, that can infect human red blood cells. Both Plasmodium falciparum and Plasmodium vivax can cause severe and fatal infections, while the other species are generally thought to be less critical and rarely result in morbidity and mortality., ${ }^{1,2}$

The emergence of multidrug resistance by the malaria parasite has significantly reduced the efficacy of most available antimalarial drugs for the treatment of malaria. There are six drug classes of antimalarial agents that are currently in use: aminoquinolines, arylaminoalcohols, artemisinins, antifolates, antibiotics and inhibitors of the respiratory chain. ${ }^{3)}$ This problem has led to the demand for new antimalarial agents with improved potential along with a greater understanding of their mechanisms of action and resistance. ${ }^{4)}$ Strains of Plasmodium falciparum have developed a remarkable degree of resistance to nearly all of the current available antimalarial drugs (e.g. chloroquine). ${ }^{5}$ ) The first antimalarial agent, lapachol, was an alkyl-1,4-naphthoquinone identified in several families of plants such as Bignoniaceae and Leguminosae. ${ }^{6}$ Inspired by the molecular structure of the lapachol, atovaquone, an alkyl substituted 2-hydroxy-1,4-naphthoquinone, has been synthesized as an antimalarial agent. ${ }^{7,8)}$ It was discovered that the atovaquone effectively inhibited plasmodial electron transport at the ubiquinone (coenzyme Q, 2) site ${ }^{9)}$ and also been determined to be an effective antimalarial drug when used to treat the multidrug-resistant malaria parasite. The discovery of this drug, which emerged from earlier studies, has provided a new approach for the exploration of bioactive molecules for fighting malaria. ${ }^{7,10)}$ Over the years, many attempts have been made towards the discovery of more potential antimalarial compounds.

During the last 50 years, various naphthoquinones and especially lawsone derivatives have been designed and extensively synthesized for the development of novel antiparasitics. ${ }^{11-14)}$ Within these studies, the 1,4-naphthoquinone analogues have been found to possess not only antimalarial activity but also a diverse range of biological activities, such as antioxidant, antibacterial, antitumor, antituberculosis activities. ${ }^{14-18)}$

Aminonaphthoquinone belongs to the quinone family which possesses an interesting scaffold conveying potential for a range of pharmacological applications..$^{19,20)}$ Previously published literature reported that incorporation of amino groups or $\mathrm{N}$-containing fragments into the 1,4-naphthoquinone skeleton resulted in increased levels of antiplasmodial activity, ${ }^{20-22)}$ as well as many other significantly beneficial biological properties such as antitumor, antifungal, antibacterial, antiplatelet, anti-allergic and anti-inflammatory activities. ${ }^{23-25)}$ Besides, 2-hydroxy-1,4-naphthoquinone analogues with the presence of 3-substituted amino groups (Fig. 1) had been shown to reveal antimalarial activity against Plasmodium falciparum. The aminomethyl linker at position 3 between the lawsone skeleton and substituents was an important counterpart for this activity. ${ }^{26)}$

Recently, ten lawsone derivatives, including 3-aryl, heteroaryl and alkyl substituted aminomethyl moieties, were synthesized by Mannich reaction. ${ }^{26)}$ Among them, five Mannich bases with aryl substituents at $R_{1}$ and $R_{2}$, displayed positive<smiles>[R7]NC([R2])C1=C(O)C(=O)c2ccccc2C1=O</smiles>

Fig. 1. Structures of 3-Substituted Aminomethyl Lawsone Derivatives 
antimalarial activity against both chloroquine (CQ)-sensitive and CQ-resistant strains of Plasmodium falciparum with $\mathrm{IC}_{50}$ values in the range of $0.41-0.50$ and $1.39-2.39 \mu \mathrm{g} / \mathrm{mL}$, respectively. However, only the naphthoquinone, possessing an alkyl ( $n$-propyl) amino group at $\mathrm{R}_{1}$, was synthesized and found to be inactive.

Because of those above reasons, we focused on the synthesis of a series of substituted aminomethyl lawsone derivatives with new side chains connected to the quinone skeleton at position 3. Furthermore, this study included an investigation of their in-vitro antimalarial activity against Plasmodium falciparum (K1, multidrug resistant strains). Consequently, our present study was consistent to the previous work by the use of the same building block of 3-substituted aminomethyl-1,4naphthoquinone. ${ }^{26)}$ It was extended further in terms of varying the substituents in order to obtain more promising antimalarial candidates. Sixteen 3-substituted-aminomethyl-2-hydroxy-1,4naphthoquinones (1-16) were prepared using a Mannich reaction to introduce two substituents at $R_{1}$ and $R_{2}$. Our target molecules can be mainly classified into two categories. In the first model, naphthoquinones 1-7 were synthesized by introduction of aliphatic substituents at $\mathrm{R}_{1}$ as $n$-butyl or allyl groups with varied substituents $(\mathrm{H}$, alkyl or aryl groups) at $\mathrm{R}_{2}$. For the second group of compounds labeled as $\mathbf{8}-\mathbf{1 6}$, they possessed aromatic substituents at $\mathrm{R}_{1}$ as dibenzyl or 4-methyl2-pyridinyl groups with many substituents $(\mathrm{H}$, alkyl or aryl groups) at $\mathrm{R}_{2}$. The structures of derivatives 1-7 and 8-16 are shown in Charts 1 and 2, respectively. As a primary objective of this research, we have focused on the synthesis of a series of 3-substituted-2-hydroxy-1,4-naphthoquinones that have new side chains connected to quinone moiety at position 3 and investigation of their in-vitro antimalarial activity against strains of Plasmodium falciparum.

\section{Results and Discussion}

The synthesis of a series of new 3-substituted derivatives of lawsone focused on new side chains connected to quinone skeleton and their in-vitro antimalarial activity against strains of Plasmodium falciparum was investigated. Synthesis of the target molecules in the first group (1-7) was achieved by Mannich reaction between 2-hydroxy-1,4-naphthoquinone and an aliphatic amine ( $n$-butyl or allyl amine) with a selected reactive aldehyde (formaldehyde or aliphatic aldehyde or aromatic aldehyde). The synthesis of derivatives 1-7 is illustrated in Chart 1.

The synthesis of the derivatives in the second group (8-16) with the pyridinylamino or dibenzylamino groups on their side chains is shown in Chart 2. Specifically, 4-methyl-2-aminopyridine and dibenzylamine were used as aromatic amines in the Mannich reaction. The reaction mechanism proceeds via two steps. In the first step, iminium ion is generated by condensation of an amine to an aldehyde. In the second step, 2-hydroxy-1,4-naphthoquinone, a resonance stabilized-carbon nucleophile, attaches itself to the iminium ion, resulting in carbon-carbon bond formation on the ring. The resulting product is identified as $\beta$-amino carbonyl compound or Mannich base. ${ }^{27)}$ In order to synthesize the 3-substituted-2-hydroxy-1,4-naphthoquinone derivatives that are associated with a variety of substituents, they must be achieved successfully through a reaction of the lawsone with various aldehydes and primary or secondary amines.<smiles>O=C1C=C(O)C(=O)c2ccccc21</smiles>

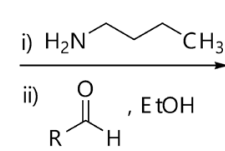<smiles>[R]C(NCCCC)C1=C(O)C(=O)c2ccccc2C1=O</smiles><smiles>[R]C([R])c1ccccc1</smiles><smiles>O=C1C=C(O)C(=O)c2ccccc21</smiles><smiles>[R]C(=O)C=CC(=O)O</smiles><smiles>[R]C(NCC=C)C1=C(O)C(=O)c2ccccc2C1=O</smiles>

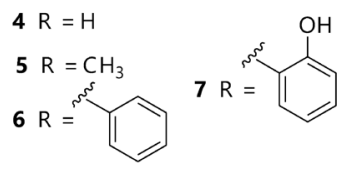

Chart 1. Synthesis of the Naphthoquinone Derivatives (1-7) with Aliphatic Substituted Amino Side Chains
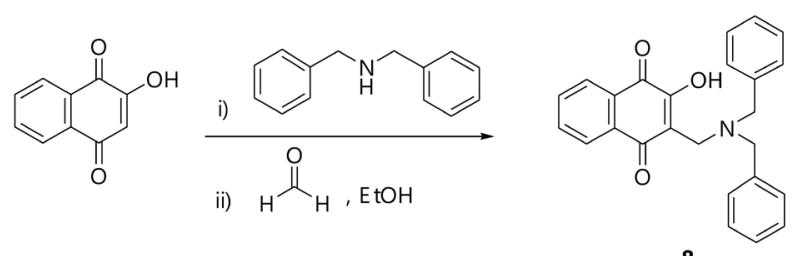<smiles>O=C1C=C(O)C(=O)c2ccccc21</smiles>
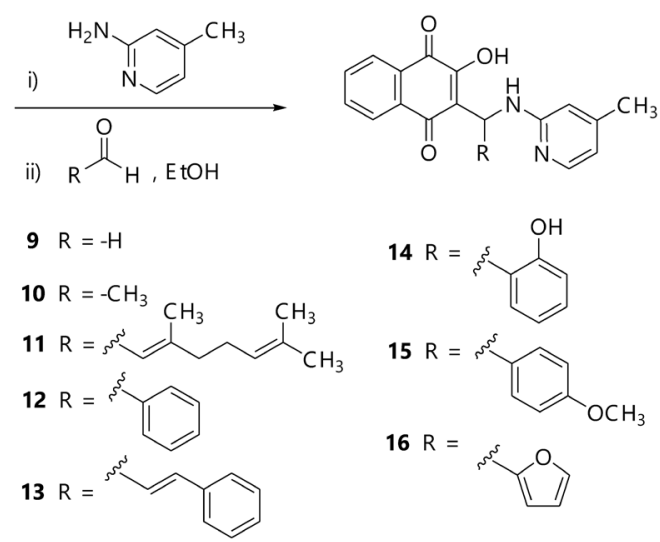

Chart 2. Synthesis of the Naphthoquinone Derivatives (8-16) with Aromatic Substituted Amino Side Chains

All derivatives were obtained in good yields (36-86\%), except for compounds $\mathbf{1}(22 \%)$ and $\mathbf{1 5}(27 \%)$. When a starting solution of starting 2-hydroxy-1,4-naphthoquinone and amine $(1: 1.1)$ in absolute ethanol was treated with selected aldehyde (refluxed at $45^{\circ} \mathrm{C}, 3 \mathrm{~h}$ ), the 3 -substituted-Mannich products were obtained. These products are highly colored as orange solids. Owing to their zwitterionic nature, the generated substances were almost insoluble. It is noteworthy to mention that the purification of all naphthoquinone derivatives failed by classical silica gel column chromatography. Due to their high polarity, they were totally adsorbed by silica gel and could not be eluted from the column. Products were successively purified by gel filtration chromatography using sephadex LH-20 as the stationary phase and by using methanol as an eluent.

Structure elucidation of all derivatives was carried out 
by ${ }^{1} \mathrm{H}-,{ }^{13} \mathrm{C}-\mathrm{NMR}$ and $2 \mathrm{D}-\mathrm{NMR}$ techniques. Their molecular formulas were confirmed by high resolution (HR)MSelectrospray ionization (ESI) data. From ${ }^{1} \mathrm{H}-\mathrm{NMR}$ spectra, all proton signals were assigned to peaks on the basis of their chemical shifts, multiplicities and coupling constants. The ${ }^{1} \mathrm{H}-\mathrm{NMR}$ spectra of all the prepared compounds primarily showed a set of characteristic signals of aromatic protons on a 2-hydroxy-1,4-naphthoquinone skeleton between 7.5-8.0 ppm as two doublets and two triplets. The hydrogen signals of the pyridine ring located on the side chain were assigned within the region of $6.5-7.8 \mathrm{ppm}$. Their ${ }^{13} \mathrm{C}-\mathrm{NMR}$ spectra were easily distinguished by the presence of quaternary carbonyl carbon signals around $181-184 \mathrm{ppm}$ and around $125-135 \mathrm{ppm}$ for the aromatic carbons of the naphthoquinone ring.

All synthetic compounds (1-16) were tested for their in-vitro antimalarial activity against stains of Plasmodium falciparum. Bioassay was performed by the microculture radioisotope technique. The $\mathrm{IC}_{50}$ values of all tested substances, as well as the positive controls (dihydroartemisinine and mefloquine) are listed in Table 1. In this regard, none of these components approached the capability of the reference drugs in terms of their inhibitory activity against Plasmodium falciparum. However, the results revealed that ten naphthoquinones $(\mathbf{1}-\mathbf{8}, \mathbf{1 1}, \mathbf{1 3})$ significantly displayed good antimalarial activity with $\mathrm{IC}_{50}$ values in the range of $0.77-4.05 \mu \mathrm{g} / \mathrm{mL}$ while the other members of the series $(9,10,12,14-16)$ were found to be inactive against the parasite. Among the tested compounds, the best antimalarial efficacy was observed in the n-butyl substituted aminomethyl lawsone derivative (1) with an $\mathrm{IC}_{50}$ value of $0.77 \mu \mathrm{g} / \mathrm{mL}$.

Modification of the substituents attached to the 1,4-naphthoquinone scaffold could be a potential factor in the modulation of antimalarial activity. Furthermore, a potential to counteract drug resistance had been observed. ${ }^{28-30)}$ Previously published literature demonstrated that amino or $N$-containing group had a significant effect on enhancing the antimalarial efficacy of the 1,4-naphthoquinone. ${ }^{20-22)}$ In the recently work, five Mannich bases with aryl substituents at $R_{1}$ and $R_{2}$ were reported to display positive antimalarial activity. ${ }^{26)}$ It was also demonstrated that naphthoquinones with aryl moieties (phenyl or $\mathrm{NO}_{2}-$, F-, OH-phenyl groups) at position 3 were more potentially important for the activity than other derivatives with heterocyclic or small alkyl groups. In addition, only the alkyl substituted naphthoquinone analogue with the three-carbon alkyl (n-propyl) group at $\mathrm{R}_{1}$ was explored and turned out to be inactive. Therefore, a series of 3-substituted aminomethyl2-hydroxy-1,4-naphthoquinone derivatives with a variety of side chains were synthesized and investigated in terms of their antiplasmodial activity.

Herein, structure-activity relationship (SAR) analysis is specifically described. All seven derivatives (1-7) possessing aliphatic side chains presented antimalarial activity whereas only three compounds $(\mathbf{8}, \mathbf{1 1}$ and 13) with aromatic substituents were found to be active against the parasite. Apparently, most naphthoquinones with alkyl and alkenyl substituted groups also exhibited greater activity when compared to the naphthoquinone analogues having aryl and heteroaryl substituted side chains. The highest activity was detected in derivative 1 , followed by 2 and 6 with $\mathrm{IC}_{50}$ values of $0.77,1.47$ and $1.63 \mu \mathrm{g} / \mathrm{mL}$, respectively. Notably, they were all members in the first category (Chart 1). It was observed that the aliphatic substituted amino group at $\mathrm{R}_{1}$ played an important role in this activity. Interestingly, all $n$-butyl substituted aminomethyl naphthoquinones (1-3) displayed effective activity whereas the similar analogue with a $n$-propyl group was previously reported as an inactive agent. ${ }^{26)}$ The elongation of the alkyl

Table 1. Antimalarial Activity ${ }^{a)}$ of the Naphthoquinone Derivatives (1-16)<smiles>[R7]NC([R2])C1=C(O)C(=O)c2ccccc2C1=O</smiles>

1-16

or $\mathrm{N}\left(\mathrm{R}_{1}\right)_{2}$ if $2^{\circ}$-amine

\begin{tabular}{cllr}
\hline \hline Compound & \multicolumn{1}{c}{$\mathrm{R}_{1}$} & $\mathrm{R}_{2}$ & Antimalarial activity IC $\mathrm{C}_{50}(\mu \mathrm{g} / \mathrm{mL})$ \\
\hline $\mathbf{1}$ & $n$-Butyl & $\mathrm{H}$ & 0.77 \\
$\mathbf{2}$ & $n$-Butyl & $\mathrm{Phenyl}$ & 1.47 \\
$\mathbf{3}$ & $n$-Butyl & 2-Hydroxyphenyl & 3.21 \\
$\mathbf{4}$ & Allyl & $\mathrm{H}$ & 4.05 \\
$\mathbf{5}$ & Allyl & Methyl & 3.28 \\
$\mathbf{6}$ & Allyl & Phenyl & 1.63 \\
$\mathbf{7}$ & Allyl & 2-Hydroxyphenyl & 3.79 \\
$\mathbf{8}$ & Dibenzyl & $\mathrm{H}$ & 3.14 \\
$\mathbf{9}$ & 4-Methyl-2-pyridinyl & $\mathrm{H}$ & Inactive \\
$\mathbf{1 0}$ & 4-Methyl-2-pyridinyl & Methyl & Inactive \\
$\mathbf{1 1}$ & 4-Methyl-2-pyridinyl & 2,6-Dimethyl-1,5-dienylheptyl & 1.83 \\
$\mathbf{1 2}$ & 4-Methyl-2-pyridinyl & Phenyl & Inactive \\
$\mathbf{1 3}$ & 4-Methyl-2-pyridinyl & Vinylphenyl & 3.15 \\
$\mathbf{1 4}$ & 4-Methyl-2-pyridinyl & 2-Hydroxyphenyl & Inactive \\
$\mathbf{1 5}$ & 4-Methyl-2-pyridinyl & 4-Methoxyphenyl & Inactive \\
$\mathbf{1 6}$ & 4-Methyl-2-pyridinyl & 2-Furanyl & Inactive \\
\hline
\end{tabular}

a) Dihydroartemisinine $\left(\mathrm{IC}_{50}=0.00153 \mu \mathrm{g} / \mathrm{mL}\right)$ and mefloquine $\left(\mathrm{IC}_{50}=0.034 \mu \mathrm{g} / \mathrm{mL}\right)$ had been used as reference drugs. 
side chain by addition of a methylene group into the $n$-propyl group led to an increase in the degree of antimalarial efficacy, suggesting that the length of the linear alkyl chain was an influential factor in facilitating this activity. Moreover, compound 11 with the presence of the citral part (a nine-carbon isoalkyl group) also showed significantly good antimalarial activity, while the bioactivity of a similar substance with a methyl substituted group (10) was no longer observed. These results corresponded to those of the previous report on atovaquone analogues (alkyl substituted-2-hydroxy-naphthoquinone derivatives) which was established that increasing the size of the branched alkyl substituents with a nine-carbon side chain enhanced inhibitory activity against the cytochrome $b c l$ complex, resulting in the most effective antiplasmodial profile. ${ }^{30,31)}$ The four-carbon saturated alkyl ( $n$-butyl), the three-carbon unsaturated alkyl (allyl) and nine-carbon isoalkyl groups on the 3 -substituted aminomethyl lawsone skeleton were more considerably predominant in terms of potent antimalarial activity in comparison to the aryl (dibenzyl) and heteroaryl (pyridinyl) substituted moieties in this study and a three-carbon saturated alkyl ( $n$-propyl) group, demonstrated in the previous report. ${ }^{26)}$

Among the aryl series, it seemed that the vinyl phenyl and benzyl groups were likely to be more essential than the 4-methy-2-aminopyridinyl and 2-furanyl groups. An observed improvement in activity in derivative $\mathbf{1 3}$ could be attributed to insertion of a vinyl group on the phenyl ring in the corresponding analogue 12. Similarly, it was determined that the allyl $\left(-\mathrm{CH}-\mathrm{CH}=\mathrm{CH}_{2}\right)$ linker at position 3 between two bulky cyclics (lawsone and phenyl rings) in compound $\mathbf{1 3}$ was a crucial requirement for this activity. Additionally, an interesting bioactivity result was also obtained from $\mathbf{8}$ with the dibenzyl group at $\mathrm{R}_{1}$, that occurred by exhibiting similarly good antimalarial activity to $\mathbf{1 3}$ with a vinyl phenyl group at $\mathrm{R}_{2}$. This outcome corresponded with the findings of the previous report which found that the aryl groups played a more vital role in this activity than the heteroaryl groups. ${ }^{26)}$ However, the attachment of $-\mathrm{OH}$ or $-\mathrm{OCH}_{3}$ to the benzene ring seemed to diminish the activity. The presence of the $\mathrm{OH}-$ phenyl moiety in 3, for example, led to an approximately 2-fold decrease in the activity with respect to $\mathbf{2}$ that was observed to bear a phenyl group. The loss of activity of the quinone derivatives 14 and 15 , which held $2-\mathrm{OH}$ or $4-\mathrm{OCH}_{3}$ phenyl substituents, respectively, were also detected.

Based on the above results, the aliphatic (n-butyl, allyl, and nine-carbon isoalkyl) side chains at position 3 seemed to be more crucial with regard to antimalarial activity than the aromatic (vinyl phenyl and dibenzyl) substituents, followed by the substituted aromatic (OH-phenyl and $\mathrm{OCH}_{3}$-phenyl) and heterocyclic aromatic (4-methy-2-aminopyridinyl and 2-furanyl) groups. Interestingly, the $n$-butyl aminomethyl moiety was observed as the most effective side chain connected to the lawsone scaffold. Importantly, variations in the substitution features of the naphthoquinone structure have a great influence on either increasing or decreasing the antiparasitic activity. Consequently, our potent antimalarial naphthoquinones have significant potential as promising antimalarial agents in the fight against malaria infections as well as in addressing the problems associated with multidrug resistance.

\section{Conclusion}

Sixteen 3-substituted methylamino lawsone derivatives (1-
16) with a variety of side chains were synthesized by Mannich reaction of the starting 2-hydroxy-1,4-naphthoquinone with selected amines and aldehydes. All components were evaluated for their in-vitro antimalarial activity against Plasmodium falciparum. Among all tested substances, ten compounds $(\mathbf{1}-\mathbf{8}, \mathbf{1 1}, \mathbf{1 3})$ showed highly active results with $\mathrm{IC}_{50}$ values in the range of $0.77-4.05 \mu \mathrm{g} / \mathrm{mL}$. The derivative 1 , processing a butylaminomethyl group as a substituent on the lawsone skeleton, displayed the strongest activity with an $\mathrm{IC}_{50}$ value of $0.77 \mu \mathrm{g} / \mathrm{mL}$. In this study, it can be concluded that the 3 -alkyl substituted amino groups on the naphthoquinone skeleton play a more vital role in this activity than the aryl or heteroaryl side chains. Chemical modification by introduction of various substituents on the lawsone scaffold has the potential to lead to potent antimalarial drugs.

\section{Experimental}

Chemistry TLC was carried out on silica gel $60 \mathrm{PF}_{254}$ precoated on an aluminium plate. Purification of synthetic products was achieved by preparative gel filtration chromatography using Sephadex LH-20 as an adsorbent. Melting points were determined on a Gallenkamp Electrothermal apparatus and were uncorrected. High resolution mass spectra were obtained from HRMS on ESI-Q-TOF-MS (Micromass, U.K.). NMR spectra were recorded at $400 \mathrm{MHz}$ on a Bruker DRX400 NMR using tetramethylsilane (TMS) as an internal standard and $\mathrm{CDCl}_{3}$ and dimethyl sulfoxide (DMSO)- $d_{6}$ as the solvents. Chemical shifts $(\delta)$ were given in parts per million (ppm) and coupling constants were recorded in Hertz (Hz). The following abbreviations are used for multiplicity: $\mathrm{s}=$ singlet, $\mathrm{br} \mathrm{s}=$ broad singlet, $\mathrm{d}=$ doublet, $\mathrm{t}=$ triplet, $\mathrm{dt}=$ doublet of triplet, $\mathrm{dd}=$ doublet of doublet, $\mathrm{ddd}=$ doublet of doublet of doublet and $m=$ multiplet.

\section{Synthesis of 1,4-Hydroquinone Derivatives}

\section{General Procedures}

The general preparation of 2-hydroxy-1,4-naphthoquinone derivatives was achieved by Mannich reaction according to an adopted procedure that had been previously reported. ${ }^{32}$ ) A mixture of the 2-hydroxy-1,4-naphthoquinone $(0.50 \mathrm{mmol})$ and an amine $(0.55 \mathrm{mmol})$ in absolute ethanol $(10 \mathrm{~mL})$ was stirred and heated at $45^{\circ} \mathrm{C}$ for $5 \mathrm{~min}$. An aldehyde $(0.55 \mathrm{mmol})$ was then added and the solution was vigorously stirred. The resulting product was obtained as a solid in $60 \mathrm{~min}$, and the suspension was stirred for a further $3 \mathrm{~h}$. Subsequently, the mixture was filtered, washed with absolute ethanol and then with diethyl ether. The filtrate was obtained as a solid and the residue was purified by preparative gel filtration chromatography. Ultimately, elution with methanol was done to afford the pure product as an orange solid.

Biological Activity The naphthoquinone derivatives (116) were subjected to in-vitro anti-malaria evaluation against Plasmodium falciparum. Bioassay was performed in the Laboratory of the National Center for Generic Engineering and Biotechnology (BIOTECH), Thailand. All naphthoquinones were tested against Plasmodium falciparum (K1, multidrug resistant strains) using the microculture radioisotope technique based upon the method described by Desjardins et al. ${ }^{33)}$

Quantitative assessment of antimalarial activity was determined as $\mathrm{IC}_{50}$, indicating that the required concentration led to reduce a $50 \%$ reduction in parasite growth. Conclusively, dihydroartemisinine (DHA) and mefloquine were used as 
reference drugs that exhibited antiplasmodial activity against Plasmodium falciparum.

Acknowledgments The authors would like to express grateful acknowledgement for the Center for Innovation in Chemistry (PERCH-CIC), Maejo University Phrae Campus and the Graduate School for financial supports. We are also thankful to the Department of Chemistry, Faculty of Science, Chiang Mai University for facilities supports.

Conflict of Interests The authors declare no conflict of interest.

Supplementary Materials The online version of this article contains supplementary materials.

\section{References}

1) Imwong M., Nakeesathit S., Day N. P. J., White N. J., Malar. J., 10, 253 (2011).

2) WHO, "World Malaria Report 2020," by WHO, Geneva, 2020.

3) Schlitzer M., ChemMedChem, 2, 944-986 (2007).

4) Grellier P., Marozienė A., Nivinskas H., Šarlauskas J., Aliverti A., Cènas N., Arch. Biochem. Biophys., 494, 32-39 (2010).

5) Panisco D. M., Keystone J. S., Drugs, 39, 160-189 (1990).

6) Hooker S. C., J. Am. Chem. Soc., 58, 1181-1190 (1936).

7) Hudson A. T., Parasitol. Today, 9, 66-68 (1993).

8) Robert A., Dechy-Cabaret O., Cazelles J., Meunier B., Acc. Chem. Res., 35, 167-174 (2002).

9) Barton V., Fisher N., Biagini G. A., Ward S. A., O'Neill P. M., Curr. Opin. Chem. Biol., 14, 440-446 (2010).

10) Fieser L. F., Berliner E., Bondhus F. J., et al., J. Am. Chem. Soc., 70, 3151-3155 (1948)

11) Prescott B., J. Med. Chem., 12, 181-182 (1969).

12) Zaugg H., Rapala I., Leffler M., J. Am. Chem. Soc., 70, 3224-3228 (1948)

13) Pingaew R., Prachayasittikul V., Worachartcheewan A., Nantasenamat C., Prachayasittikul S., Ruchirawat S., Prachayasittikul V., Eur. J. Med. Chem., 103, 446-459 (2015).

14) Aminin D., Polonik S., Chem. Pharm. Bull., 68, 46-57 (2020).

15) Deniz N. G., Ibis C., Gokmen Z., Stasevych M., Novikov V., Komarovska-Porokhnyavets O., Ozyurek M., Guclu K., Karakas D.,
Ulukaya E., Chem. Pharm. Bull., 63, 1029-1039 (2015).

16) Choudhari D., Lande D. N., Chakravarty D., Gejji S. P., Das P., Pardesi K. R., Satpute S., Salunke-Gawali S., J. Mol. Struct., 1176, 194-206 (2019).

17) Kongkathip N., Kongkathip B., Siripong P., Sangma C., Luangkamin S., Niyomdecha M., Pattanapa S., Piyaviriyagulb S., Kongsaeree P., Bioorg. Med. Chem., 11, 3179-3191 (2003).

18) Paengsri W., Baramee A., Chiang Mai J. Sci., 40, 70-76 (2013).

19) Benites J., Valderrama J. A., Bettega K., Pedrosa R. C., Calderon P. B., Verrax J., J. Med. Chem., 45, 6052-6057 (2010).

20) Kapadia G. J., Azuine M. A., Balasubramanian V., Sridhar R., Pharmacol. Res., 43, 363-367 (2001).

21) Fiorot R. G., Allochio Filho J. F., Pereira T. M. C., Lacerda V. Jr., dos Santos R. B., Romão W., Greco S. J., Tetrahedron Lett., 55, 4373-4377 (2014).

22) Leffler M. T., Hathaway R. J., J. Am. Chem. Soc., 70, 3222-3223 (1948).

23) Vargas M. D., Pinto A. C., Echevarria A., Esteves-Souza A., Camara C. A., Cunha A. C., Torres J. C., Lima E. L. S., J. Braz. Chem. Soc., 17, 439-442 (2006).

24) Tandon V. K., Yadav D. B., Singh R. V., Chaturvedi A. K., Shukla P. K., Bioorg. Med. Chem. Lett., 15, 5324-5328 (2005).

25) Lien J., Huang L., Wang J., Teng C., Lee K., Kuo S., Chem. Pharm. Bull., 44, 1181-1187 (1996).

26) Arundhati M., Dipak C., Mithun R., Indian J. Pharm. Educ. Res., 52, 472-479 (2018)

27) Akiyama T., "Comprehensive Organic Synthesis II," 2nd ed., Vol. 2, Elsevier, the Netherlands, 2014, pp. 629-681.

28) Hage S. E., Ane M., Stigliani J., Marjorie M., Vial H., BaziardMouysset G., Payard M., Eur. J. Med. Chem., 44, 4778-4782 (2009).

29) Hughes L., Lanteri C., O’Neil M., Johnson J., Gribble G., Trumpower B., Mol. Biochem. Parasitol., 177, 12-19 (2011).

30) Fieser L. J., Schirmer J. P., Archer S., Lorenz R. R., Pfaffenbach P. I., J. Med. Chem., 10, 513-517 (1967).

31) Kessl J. J., Moskalev N. V., Gribble G. W., Nasr M., Meshnick S. R., Trumpower B. L., Biochim. Biophys. Acta, 1767, 319-326 (2007).

32) Baramee A., Coppin A., Mortuaire M., Pelinski L., Tomavo S., Brocard J., Bioorg. Med. Chem., 14, 1294-1302 (2006).

33) Desjardins R. E., Canfield C. J., Haynes J. D., Chulay J. D., Antimicrob. Agents Chemother., 16, 710-718 (1979). 